\title{
Blow-up Phenomena and Persistence Properties of Solutions to the Two-Component DGH Equation
}

\author{
Panpan Zhai, Zhengguang Guo, and Weiming Wang \\ College of Mathematics and Information Science, Wenzhou University, Wenzhou 325035, China \\ Correspondence should be addressed to Weiming Wang; weimingwang2003@163.com
}

Received 11 April 2013; Accepted 22 May 2013

Academic Editor: Carlo Bianca

Copyright (C) 2013 Panpan Zhai et al. This is an open access article distributed under the Creative Commons Attribution License, which permits unrestricted use, distribution, and reproduction in any medium, provided the original work is properly cited.

\begin{abstract}
This paper is concerned with blow-up phenomena and persistence properties for an integrable two-component Dullin-GottwaldHolm shallow water system. We give sufficient conditions on the initial data which guarantee blow-up phenomena of solutions in finite time for both periodic and nonperiodic cases, respectively. Furthermore, the persistence properties of solutions to the system are investigated.
\end{abstract}

\section{Introduction}

In 2001, Dullin et al. [1] derived a new equation by using the method of asymptotic analysis and a near-identity normal form transformation in water wave theory to describe the unidirectional propagation of surface waves in a shallow water regime, it reads as follows:

$$
m_{t}+2 \omega u_{x}+u m_{x}+2 m u_{x}=-\gamma u_{x x x}, \quad x \in \mathbb{R}, t>0 .
$$

Since (1) was derived by Dullin et al., we call it DGH equation. Let $m=u-\alpha^{2} u_{x x}$; then we can rewrite (1) as the following form:

$$
u_{t}-\alpha^{2} u_{x x t}+2 \omega u_{x}+3 u u_{x}+\gamma u_{x x x}=\alpha^{2}\left(2 u_{x} u_{x x}+u u_{x x x}\right),
$$

where $\alpha^{2}$ and $\gamma / 2 \omega$ are squares of length scales, the constant $\omega=\sqrt{g h} / 2$ is the critical shallow water speed for undisturbed water at rest at spatial infinity, $h$ is the mean fluid depth, and $g$ is the gravitational constant. This equation is connected with two separately integrable soliton equations for shallow water waves.

When $\alpha^{2}=0$, (2) becomes the KdV equation as follows:

$$
u_{t}+2 \omega u_{x}+3 u u_{x}=-\gamma u_{x x x} .
$$

Bourgain proved that solutions to the $\mathrm{KdV}$ equation are global as long as the initial data is square integrable [2], which is also shown in [3]. Another remarkable property is that it is integrable and the solitary waves are nonlinearly stable, and when $\omega=0$, there exists a smooth soliton solution [4].

Instead, taking $\gamma=0$ in (2), it turns out to be the CamassaHolm equation:

$$
u_{t}-\alpha^{2} u_{x x t}+2 \omega u_{x}+3 u u_{x}=\alpha^{2}\left(2 u_{x} u_{x x}+u u_{x x x}\right),
$$

which was derived by Camassa and Holm in [5] by approximating directly the Hamiltonian for Euler's equations in the shallow water regime in 1993. Many researches have been carried out on the Camassa-Holm equation in recent years. Local well-posedness has been proven by several authors; see [6-9]. Blow-up phenomena have been investigated in [10-15]. Besides, in [11], global solutions were also discussed. In [16], global existence of weak solutions was proved, but uniqueness was obtained only under a priori assumption. Furthermore, the authors in [17] studied the persistence properties of solution to the Camassa-Holm equation. In [1820], the soliton solution was studied.

It is very interesting that (2) still preserves the biHamiltonian structure and complete integrability. Indeed, (2) can be rewritten in two compatible Hamiltonian forms in terms of $m=u-\alpha^{2} u_{x x}[1]$ as follows,

$$
m_{t}=-B_{2} \frac{\delta E}{\delta m}=-B_{1} \frac{\delta F}{\delta m}
$$


where

$$
\begin{gathered}
B_{1}=\partial_{x}-\alpha^{2} \partial_{x}^{3}, \\
B_{2}=\partial_{x}(m+\omega)+(m+\omega) \partial_{x}+\gamma \partial_{x}^{3}, \\
E(u)=\frac{1}{2} \int\left(u^{2}+\alpha^{2} u_{x}^{2}\right) d x, \\
F(u)=\frac{1}{2} \int\left(u^{3}+\alpha^{3} u u_{x}^{2}+2 \omega u^{2}-\gamma u_{x}^{2}\right) d x .
\end{gathered}
$$

$E(u)$ and $F(u)$ are two conserved quantities.

In [21], Tian et al. studied the well-posedness of the Cauchy problem and the scattering problem of the DGH equation. The issue of passing to the limit as the dispersive parameter tends to zero for the solution of the DGH equation was investigated, and the convergence of the solutions to DGH equation as $\alpha^{2} \rightarrow 0$ was studied. Besides, data of the scattering problem for the equation could be explicitly expressed. The blow-up phenomenon of solutions to the DGH equation was the subject of [21-24].

In this paper, we are interested in the following Cauchy problem for an integrable two-component DGH equation. It reads as

$$
\begin{gathered}
u_{t}-\alpha^{2} u_{t x x}+2 \omega u_{x}+3 u u_{x}+\gamma u_{x x x} \\
=\alpha^{2}\left(2 u_{x} u_{x x}+u u_{x x x}\right)-\sigma \bar{\rho} \bar{\rho}_{x}, \quad x \in \mathbb{R}, t>0, \\
\bar{\rho}_{t}+(\bar{\rho} u)_{x}=0, \quad x \in \mathbb{R}, t>0 \\
u(0, x)=u_{0}(x), \quad x \in \mathbb{R} \\
\bar{\rho}(0, x)=\bar{\rho}_{0}(x), \quad x \in \mathbb{R} .
\end{gathered}
$$

The variable $u(x, t)$ describes the horizontal velocity of the fluid, and $\bar{\rho}(x, t)$ denotes the horizontal deviation of the surface from equilibrium, all measured in dimensionless units. This model can be derived by Constantin and Ivanov's approach [25] from shallow water theory, which includes the two-component Camassa-Holm system [26-32] as its special case with $\alpha=1$ and $\gamma=0$ in (7). From a geometric point of view, (7) is the model for geodesic motion on the semidirect product Lie group of diffeomorphisms acting on densities, with respect to the $H^{1}$-norm of velocity and the $L^{2}$-norm on the density. Mathematically, (7) admits not only breakingwave solutions but also global in time solutions. In view of the context of hydrodynamics, we have the assumptions $u \rightarrow 0$, $\bar{\rho} \rightarrow 1$ as $x \rightarrow \infty$, at any instant $t$. For convenience of later discussion, letting $\rho=\bar{\rho}-1, \alpha>0$, and $\sigma=1$, we have $\rho \rightarrow 0$ as $x \rightarrow \infty$. Then it follows that

$$
\begin{gathered}
u_{t}-\alpha^{2} u_{t x x}+2 \omega u_{x}+3 u u_{x}+\gamma u_{x x x}-2 \alpha^{2} u_{x} u_{x x} \\
-\alpha^{2} u u_{x x x}+\rho \rho_{x}+\rho_{x}=0, \quad x \in \mathbb{R}, t>0, \\
\rho_{t}+(\rho u)_{x}+u_{x}=0, \quad x \in \mathbb{R}, t>0,
\end{gathered}
$$

$$
\begin{array}{ll}
u(0, x)=u_{0}(x), & x \in \mathbb{R}, \\
\rho(0, x)=\rho_{0}(x), & x \in \mathbb{R} .
\end{array}
$$

Define $\Lambda=\left(1-\alpha^{2} \partial_{x}^{2}\right)^{-1}$; then the operator $\Lambda$ can be expressed by

$$
\Lambda f=G * f=\int_{\mathbb{R}} G(x-y) f(y) d y,
$$

where $G(x)$ is the Green function for $\Lambda$ and * denotes the convolution. It is easy to get $G *(1 / 2)=1 / 2$. For the periodic case, $G(x)=\cosh ((x / \alpha)-([x] / \alpha)-(1 / 2 \alpha)) / 2 \alpha \sinh (1 / 2 \alpha)$ is the Green function for $\Lambda$ in the unit circle. While, for the nonperiodic case, the Green function for $\Lambda$ in $\mathbb{R}$ is $G(x)=$ $(1 / 2 \alpha) e^{-|x / \alpha|}$. Using this identity, we can rewrite (1) as the following nonlocal form:

$$
\begin{aligned}
& u_{t}+u_{x}\left(u-\frac{\gamma}{\alpha^{2}}\right) \\
&=-\partial_{x} G *\left(u^{2}+\frac{\alpha^{2}}{2} u_{x}^{2}+\left(2 \omega+\frac{\gamma}{\alpha^{2}}\right) u+\frac{1}{2} \rho^{2}+\rho\right), \\
& x \in \mathbb{R}, t>0, x \in \mathbb{R}, t>0, \\
& \rho_{t}+(\rho u)_{x}+u_{x}=0, \quad x \in \mathbb{R}, \\
& u(0, x)=u_{0}(x), \quad x \in \mathbb{R} . \\
& \rho(0, x)=\rho_{0}(x), \quad x
\end{aligned}
$$

We note that less deep results exist yet for this model except for the recent ones $[33,34]$. Our motivation here is to explore some new blow-up conditions especially for the initial data were added on some different quantities. This paper is organized as follows. In Section 2, we recall some preliminary results on local well-posedness and blow-up scenario and present some useful conversation laws. In Section 3, we study the blow-up phenomenon of strong solutions in both periodic and nonperiodic cases. In Section 4, persistence properties of solutions to the system are studied.

\section{Preliminaries}

We can use Kato's theory [35] to establish the following local well-posedness theorem for (10); its proof is referred to in the discussions of $[33,34]$, so we omit it here.

Theorem 1. Assume an initial data $\left(u_{0}, \rho_{0}\right) \in H^{s} \times H^{s-1}, s \geq$ 2. Then there exists a maximal $T=T\left(\left\|\left(u_{0}, \rho_{0}\right)\right\|_{H^{s} \times H^{s-1}}\right)>0$ and a unique solution

$$
(u, \rho) \in C\left([0, T) ; H^{s} \times H^{s-1}\right) \cap C^{1}\left([0, T) ; H^{s-1} \times H^{s-2}\right)
$$

of system (10). Moreover, the solution $(u, \rho)$ depends continuously on the initial value $\left(u_{0}, \rho_{0}\right)$, and the maximal time of existence $T>0$ is independent of $s$.

Next, we will give some useful conserved quantities, which are important to discuss the blow-up criteria. 
Proposition 2. Suppose $\left(u_{0}, \rho_{0}\right) \in H^{s} \times H^{s-1}, s \geq 2$; then the solution $(u, \rho)$ of the system (10) guaranteed by Theorem 1 satisfies

$$
\int_{\mathbb{R}}\left(u^{2}+\alpha^{2} u_{x}^{2}+\rho^{2}\right) d x=\int_{\mathbb{R}}\left(u_{0}^{2}+\alpha^{2} u_{0 x}^{2}+\rho_{0}^{2}\right) d x .
$$

Proof. Consider

$$
\begin{aligned}
& \frac{d}{d t} \int_{\mathbb{R}}\left(u^{2}+\alpha^{2} u_{x}^{2}+\rho^{2}\right) d x \\
& \quad=\int_{\mathbb{R}}\left(2 u u_{t}+2 \alpha^{2} u_{x} u_{x t}+2 \rho \rho_{t}\right) d x \\
& \quad=\int_{\mathbb{R}}\left[2 u\left(u_{t}-\alpha^{2} u_{x x t}\right)+2 \rho \rho_{t}\right] d x .
\end{aligned}
$$

From the first equation of (10), we have

$$
\begin{aligned}
\int_{\mathbb{R}} 2 u\left(u_{t}-\alpha^{2} u_{x x t}\right) d x & \\
=\int_{\mathbb{R}} 2 u( & -2 \omega u_{x}-3 u u_{x}-\gamma u_{x x x} \\
& \left.+2 \alpha^{2} u_{x} u_{x x}+\alpha^{2} u u_{x x x}-\rho \rho_{x}-\rho_{x}\right) d x .
\end{aligned}
$$

Similarly, from the second equation of (10), we can get

$$
\int_{\mathbb{R}} 2 \rho \rho_{t} d x=\int_{\mathbb{R}}-2 \rho\left((\rho u)_{x}+u_{x}\right) d x .
$$

So we can obtain

$$
\begin{aligned}
& \frac{d}{d t} \int_{\mathbb{R}}\left(u^{2}+\alpha^{2} u_{x}^{2}+\rho^{2}\right) d x \\
& =\int_{\mathbb{R}}\left[2 u \left(-2 \omega u_{x}-3 u u_{x}-\gamma u_{x x x}+2 \alpha^{2} u_{x} u_{x x}\right.\right. \\
& \left.\left.\quad+\alpha^{2} u u_{x x x}-\rho \rho_{x}-\rho_{x}\right)-2 \rho\left((\rho u)_{x}+u_{x}\right)\right] d x \\
& =\int_{\mathbb{R}}\left[-2 \omega\left(u^{2}\right)_{x}-2\left(u^{3}\right)_{x}+\gamma\left(u_{x}^{2}\right)_{x}\right. \\
& \left.\quad+2 \alpha^{2}\left(u^{2} u_{x x}\right)_{x}-2(\rho u)_{x}-2(\rho \rho u)_{x}\right] d x \\
& =0 .
\end{aligned}
$$

This completes the proof.

We also note that the quantities $\int_{\mathbb{R}} u(x, t) d x$ and $\int_{\mathbb{R}} \rho(x, t) d x$ are also conserved for $\rho_{0}(x) \in L^{1}, u_{0}(x) \in$ $L^{1}$. These conservation laws will play important roles in considering the behavior of solutions. In what follows, we denote $\int_{\mathbb{R}}\left(u^{2}+\alpha^{2} u_{x}^{2}+\rho^{2}\right) d x$ by $\|(u, \rho)\|_{H^{1} \times L^{2}}^{2}$.

Moreover, using the techniques in [36], one can get the following criterion for finite time wave breaking to (10).

Theorem 3 (see [34]). Let $\left(u_{0}, \rho_{0}\right) \in H^{s} \times H^{s-1}$ with $s \geq 2$, and let $T>0$ be the maximal time of existence of the solution $(u, \rho)$ to the model $(10)$ with initial data $\left(u_{0}, \rho_{0}\right)$. Then the corresponding solution $(u, \rho)$ blows up in finite time if and only if

$$
\lim _{t \uparrow T}\left\{\inf _{x \in \mathbb{R}} u_{x}(t, x)\right\}=-\infty
$$

We will then need to introduce the standard particle trajectory method for later use. Now consider the following initial value problem:

$$
\begin{gathered}
q_{t}=u(t, q), \quad t \in[0, T), \\
q(0, x)=x, \quad x \in \mathbb{R},
\end{gathered}
$$

where $u \in C^{1}\left([0, T), H^{s-1}\right)$ is the first component of the solution $(u, \rho)$ to system $(10)$ with initial data $\left(u_{0}, \rho_{0}\right) \in H^{s} \times$ $H^{s-1},(s \geq 2)$, and $T>0$ is the maximal time of existence. By direct calculation, we have

$$
q_{t x}(t, x)=u_{x}(t, q(t, x)) q_{x}(t, x) .
$$

Then,

$$
q_{x}(t, x)=\exp \left(\int_{0}^{t} u_{x}(\tau, q(\tau, x)) d \tau\right)>0, \quad t>0, x \in \mathbb{R},
$$

which means that $q(t, \cdot): \mathbb{R} \rightarrow \mathbb{R}$ is a diffeomorphism of the line for every $t \in[0, T)$. Consequently, the $L^{\infty}$-norm of any function $v(t, \cdot)$ is preserved under the family of the diffeomorphism $q(t, \cdot)$; that is,

$$
\|v(t, \cdot)\|_{L^{\infty}}=\|v(t, q(t, \cdot))\|_{L^{\infty}}, \quad t \in[0, T) .
$$

Similarly,

$$
\begin{array}{ll}
\inf _{x \in \mathbb{R}} v(t, x)=\inf _{x \in \mathbb{R}} v(t, q(t, x)), & t \in[0, T), \\
\sup _{x \in \mathbb{R}} v(t, x)=\sup _{x \in \mathbb{R}} v(t, q(t, x)), & t \in[0, T) .
\end{array}
$$

\section{Blow-Up Criteria}

In this section, we establish sufficient conditions to guarantee the formation of singularities for the corresponding solutions to (10) in periodic and nonperiodic cases. These sufficient conditions are different from each other.

Hereinafter, we investigate the blow-up phenomenon with periodic setting; that is, $x \in \mathbb{S}=\mathbb{R} / \mathbb{Z}$. First, we introduce the following lemmas for later use.

Lemma 4 (see [23]). For all $f \in H^{1}(\mathbb{S})$, the following inequality holds:

$$
G *\left(f^{2}+\frac{\alpha^{2}}{2} f_{x}^{2}\right)(x) \geq C_{0} f^{2}(x)
$$


with

$C_{0}$

$$
=\frac{1}{2}+\frac{\arctan (\sinh (1 / 2 \alpha))}{2 \sinh (1 / 2 \alpha)+2 \arctan (\sinh (1 / 2 \alpha)) \sinh ^{2}(1 / 2 \alpha)} .
$$

Moreover, $C_{0}$ is the optimal constant obtained by the function

$$
\begin{aligned}
f_{0}=( & 1+\arctan \left(\sinh \left(\frac{x}{\alpha}-\frac{[x]}{\alpha}-\frac{1}{2 \alpha}\right)\right) \\
& \left.\times \sinh \left(\frac{x}{\alpha}-\frac{[x]}{\alpha}-\frac{1}{2 \alpha}\right)\right) \\
& \times\left(1+\arctan \left(\sinh \left(\frac{1}{2 \alpha}\right)\right) \sinh \left(\frac{1}{2 \alpha}\right)\right)^{-1}
\end{aligned}
$$

Lemma 5 (see [23]). For all $f \in H^{1}(\mathbb{S})$, the following inequality holds:

$$
\max _{x \in \mathbb{S}} f^{2}(x) \leq C_{1} \int_{\mathbb{S}}\left(f^{2}+\alpha^{2} f_{x}^{2}\right) d x
$$

where

$$
C_{1}=\frac{\cosh (1 / 2 \alpha)}{2 \alpha \sinh (1 / 2 \alpha)}
$$

Moreover, $C_{1}$ is the minimum value, so in this sense, $C_{1}$ is the optimal constant which is obtained by the associated Green function

$$
G(x)=\frac{\cosh ((x / \alpha)-([x] / \alpha)-(1 / 2 \alpha))}{2 \alpha \sinh (1 / 2 \alpha)}
$$

Lemma 6 (see [37]). Assume that a differentiable function $y(t)$ satisfies

$$
y^{\prime}(t) \leq-C y^{2}(t)+K
$$

with the constants $C, K>0$. If the initial datum $y(0)=y_{0}<$ $-\sqrt{K / C}$, then the solution to the previous equation goes to $-\infty$ in finite time.

Lemma 7 (see [24]). Assume $f(x) \in H^{s}(\mathbb{S}), s>2$. If $\int_{\mathbb{S}} f(x) d x=0$, then

$$
\begin{gathered}
\|f(x)\|_{L^{\infty}(\mathbb{S})}^{2} \leq \frac{1}{12} \int_{\mathbb{S}} f_{x}^{2}(x) d x \\
\int_{\mathbb{S}} f^{2}(x) d x \leq \frac{1}{12} \int_{\mathbb{S}} f_{x}^{2}(x) d x \\
\int_{\mathbb{S}} f^{2}(x) f_{x}^{2}(x) d x \leq \frac{1}{12}\left(\int_{\mathbb{S}} f_{x}^{2}(x) d x\right)^{2} .
\end{gathered}
$$

Now we give some sufficient conditions on the initial data to guarantee finite time wave breaking for the periodic case.
Theorem 8. Assume that the initial data $\left(u_{0}, \rho_{0}\right) \in H^{s} \times$ $H^{s-1}, s \geq 2$, satisfies

$$
\int_{\mathbb{S}} u_{0 x}^{3} d x<-\frac{\left\|\left(u_{0}, \rho_{0}\right)\right\|_{H_{\alpha}^{1} \times L^{2}}^{2}}{\alpha^{3}} \sqrt{3 A+6 C_{1}\left\|\left(u_{0}, \rho_{0}\right)\right\|_{H_{\alpha}^{1} \times L^{2}}^{2}}
$$

where $C_{1}$ is defined as in Lemma 5, and constant $A$ is determined later. Moreover, for convenience, we suppose that $2 \omega+\gamma / \alpha^{2}=0$. Then the corresponding strong solution to (10) blows up in finite time.

Proof. By assumption and Theorem 1, there exists a solution $(u, \rho)$ to system $(10)$ corresponding to the initial value $\left(u_{0}, \rho_{0}\right) \in H^{s} \times H^{s-1}, s \geq 2$. Suppose that the statement is not true; that is, the corresponding solution does not blow up in finite time, and then due to Theorem 3 , there exists $M_{1} \geq 0$, such that

$$
\inf _{(t, x) \in[0, T) \times \mathbb{R}} u_{x}(t, x) \geq-M_{1}
$$

Let

$$
\begin{array}{r}
M(t)=u_{x}(t, q(t, x)), \quad \gamma(t)=\rho(t, q(t, x)), \\
t \in[0, T),
\end{array}
$$

with $q(t, x)$ determined in (18). Then we have the following inequality for each $x \in \mathbb{R}$ and $t \in[0, T)$ :

$$
M(t) \geq-M_{1} \text {. }
$$

Since

$$
\gamma^{\prime}(t)=-\gamma(t) M(t),
$$

we have, by solving it,

$$
|\gamma(t)|=|\gamma(0)| e^{\int_{0}^{t}-M(\tau) d \tau}
$$

So we can get

$$
|\rho(t, q(t, x))|=|\gamma(t)|=|\gamma(0)| e^{\int_{0}^{t}-M(\tau) d \tau} \leq\left\|\rho_{0}\right\|_{L^{\infty}} e^{M_{1} t},
$$

then we have

$$
\|\rho(t, \cdot)\|_{L^{\infty}} \leq\left\|\rho_{0}\right\|_{L^{\infty}} e^{M_{1} t} .
$$

Therefore, the following inequality holds:

$$
\left\|(\rho+1)^{2}\right\|_{L^{\infty}} \leq\left(\|\rho\|_{L^{\infty}}+1\right)^{2} \leq\left(\left\|\rho_{0}\right\|_{L^{\infty}} e^{M_{1} t}+1\right)^{2} \doteq A .
$$

With the notation of $\partial_{x}^{2}(G * f)=\left(1 / \alpha^{2}\right)(G * f-f)$, differentiating both sides of the first equation of (10) with respect to variable $x$, we obtain

$$
\begin{aligned}
u_{t x} & +u_{x}^{2}+u u_{x x}+2 \omega u_{x x} \\
= & \frac{1}{\alpha^{2}}\left(u^{2}+\frac{\alpha^{2}}{2} u_{x}^{2}+\frac{1}{2} \rho^{2}+\rho\right) \\
& \quad-\frac{1}{\alpha^{2}} G *\left(u^{2}+\frac{\alpha^{2}}{2} u_{x}^{2}+\frac{1}{2} \rho^{2}+\rho\right) .
\end{aligned}
$$


We note that $G *(1 / 2)=1 / 2$; then $(40)$ can be rewritten as follows:

$$
\begin{aligned}
u_{t x}+ & u_{x}^{2}+u u_{x x}+2 \omega u_{x x} \\
= & \frac{1}{\alpha^{2}}\left(u^{2}+\frac{\alpha^{2}}{2} u_{x}^{2}+\frac{1}{2}(\rho+1)^{2}\right) \\
& \quad-\frac{1}{\alpha^{2}} G *\left(u^{2}+\frac{\alpha^{2}}{2} u_{x}^{2}+\frac{1}{2}(\rho+1)^{2}\right) .
\end{aligned}
$$

Multiplying $u_{x}^{2}$ on both sides and integrating by parts with respect to $x$, one obtains

$$
\begin{aligned}
\frac{d}{d t} \int_{\mathbb{S}} u_{x}^{3} d x \\
=-\frac{1}{2} \int_{\mathbb{S}} u_{x}^{4} d x+\frac{3}{\alpha^{2}} \int_{\mathbb{S}} u_{x}^{2} u^{2} d x \\
\quad+\frac{3}{\alpha^{2}} \int_{\mathbb{S}} u_{x}^{2}\left(\frac{1}{2}(\rho+1)^{2}\right) d x \\
\quad-\frac{3}{\alpha^{2}} \int_{\mathbb{S}} u_{x}^{2} G *\left(u^{2}+\frac{\alpha^{2}}{2} u_{x}^{2}+\frac{1}{2}(\rho+1)^{2}\right) d x \\
\leq-\frac{1}{2} \int_{\mathbb{S}} u_{x}^{4} d x+\frac{3}{\alpha^{2}} \int_{\mathbb{S}} u_{x}^{2} u^{2} d x \\
+\frac{3}{\alpha^{2}} \int_{\mathbb{S}} u_{x}^{2}\left(\frac{1}{2}(\rho+1)^{2}\right) d x,
\end{aligned}
$$

where we have used the following identity:

$$
\int_{\mathbb{S}} u_{x}^{2} u u_{x x} d x=-\frac{1}{3} \int_{\mathbb{S}} u_{x}^{4} d x
$$

Since

$$
\left(\int_{\mathbb{S}} u_{x}^{3} d x\right)^{2} \leq \frac{1}{\alpha^{2}}\left(\int_{\mathbb{S}} u_{x}^{4} d x\right)\left(\int_{\mathbb{S}} \alpha^{2} u_{x}^{2} d x\right),
$$

it follows that

$$
\int_{\mathbb{S}} u_{x}^{4} d x \geq \frac{\alpha^{2}}{\left\|\left(u_{0}, \rho_{0}\right)\right\|_{H_{\alpha}^{1} \times L^{2}}^{2}}\left(\int_{\mathbb{S}} u_{x}^{3} d x\right)^{2} .
$$

We can also get

$$
\begin{aligned}
& \frac{3}{\alpha^{2}} \int_{\mathbb{S}} u_{x}^{2} u^{2} d x \leq \frac{3}{\alpha^{4}} C_{1}\left\|\left(u_{0}, \rho_{0}\right)\right\|_{H_{\alpha}^{1} \times L^{2}}^{4}, \\
& \left|\frac{3}{\alpha^{2}} \int_{\mathbb{S}} u_{x}^{2}\left(\frac{1}{2}(\rho+1)^{2}\right) d x\right| \\
& \quad=\frac{3}{2 \alpha^{2}} \int_{\mathbb{S}} u_{x}^{2}(\rho+1)^{2} d x \\
& \leq \frac{3}{2 \alpha^{4}}\left\|(\rho+1)^{2}\right\|_{L^{\infty}} \int_{\mathbb{S}} \alpha^{2} u_{x}^{2} d x \\
& \leq \frac{3}{2 \alpha^{4}}\left\|(\rho+1)^{2}\right\|_{L^{\infty}}\left\|\left(u_{0}, \rho_{0}\right)\right\|_{H_{\alpha}^{1} \times L^{2}}^{2}
\end{aligned}
$$

So we obtain

$$
\begin{aligned}
& \frac{d}{d t} \int_{\mathbb{S}} u_{x}^{3} d x \\
& \leq-\frac{\alpha^{2}}{2\left\|\left(u_{0}, \rho_{0}\right)\right\|_{H_{\alpha}^{1} \times L^{2}}^{2}}\left(\int_{\mathbb{S}} u_{x}^{3} d x\right)^{2}+\frac{3}{\alpha^{4}} C_{1}\left\|\left(u_{0}, \rho_{0}\right)\right\|_{H_{\alpha}^{1} \times L^{2}}^{4} \\
& \quad+\frac{3}{2 \alpha^{4}}\left\|(\rho+1)^{2}\right\|_{L^{\infty}}\left\|\left(u_{0}, \rho_{0}\right)\right\|_{H_{\alpha}^{1} \times L^{2}}^{2} \\
& \leq-\frac{\alpha^{2}}{2\left\|\left(u_{0}, \rho_{0}\right)\right\|_{H_{\alpha}^{1} \times L^{2}}^{2}}\left(\int_{\mathbb{S}} u_{x}^{3} d x\right)^{2}+\frac{3}{\alpha^{4}} C_{1}\left\|\left(u_{0}, \rho_{0}\right)\right\|_{H_{\alpha}^{1} \times L^{2}}^{4} \\
& \quad+\frac{3}{2 \alpha^{4}} A\left\|\left(u_{0}, \rho_{0}\right)\right\|_{H_{\alpha}^{1} \times L^{2}}^{2} .
\end{aligned}
$$

Due to the theory of Riccati type differential equations in Lemma 6, if (31) holds, then we have

$$
\int_{\mathbb{S}} u_{x}^{3} d x \rightarrow-\infty
$$

as $t$ tends to some $T_{0}$.

On the other hand, the majorization

$$
\begin{aligned}
\int_{\mathbb{S}} u_{x}^{3} d x & \geq \inf u_{x}(x, t) \int_{\mathbb{S}} u_{x}^{2} d x \\
& >c(\alpha) \inf u_{x}(x, t)\left\|\left(u_{0}, \rho_{0}\right)\right\|_{H_{\alpha}^{1} \times L^{2}}^{2}
\end{aligned}
$$

implies that

$$
\lim _{t \uparrow T_{0}} \inf u_{x}(x, t)=-\infty .
$$

It contradicts the assumption

$$
\inf _{(t, x) \in[0, T) \times \mathbb{R}} u_{x}(t, x) \geq-M_{1} .
$$

By Theorem 3, we know that the solution must blow up in finite time.

Next, we find if the initial velocity has zero mean and initial energy is sufficiently large, wave breaking can occur.

Theorem 9. Suppose $z_{0}=\left(u_{0}, \rho_{0}\right) \in H^{s} \times H^{s-1}, s>5 / 2$, and $\alpha$ satisfies $0<\sinh (1 / 2 \alpha) / \alpha<3$. If the initial energy satisfies $\int_{\mathbb{S}}\left(u_{0}^{2}+\alpha^{2} u_{0 x}^{2}+\rho_{0}^{2}\right) d x>C_{0}$ for some positive constant $C_{0}$, and then $\int_{\mathbb{S}} u_{0}(x) d x=0$ holds for nontrival $z_{0}$. Besides, assume that $2 \omega+\gamma / \alpha^{2}=0$ just for convenience. Then the solution to the system (10) with initial value $z_{0}$ blows up in finite time.

Proof. From the first equation of (10), we know that

$$
\begin{aligned}
\frac{d}{d t} \int_{\mathbb{S}} & u_{x}^{3} d x \\
= & -\frac{1}{2} \int_{\mathbb{S}} u_{x}^{4} d x+\frac{3}{\alpha^{2}} \int_{\mathbb{S}} u_{x}^{2} u^{2} d x+\frac{3}{\alpha^{2}} \int_{\mathbb{S}} u_{x}^{2}\left(\frac{1}{2} \rho^{2}+\rho\right) d x \\
& -\frac{3}{\alpha^{2}} \int_{\mathbb{S}} u_{x}^{2} G *\left(u^{2}+\frac{\alpha^{2}}{2} u_{x}^{2}+\frac{1}{2} \rho^{2}+\rho\right) d x
\end{aligned}
$$


On the other hand, we know that $\int_{\mathbb{S}} u(x, t) d x=0$ in view of the hypothesis, and the following inequality holds:

$$
\frac{1}{2 \alpha \sinh (1 / 2 \alpha)} \leq G(x) \leq \frac{\cosh (1 / 2 \alpha)}{2 \alpha \sinh (1 / 2 \alpha)} \text {. }
$$

Using Lemma 7 and (53), we obtain

$$
\begin{aligned}
\frac{d}{d t} \int_{\mathbb{S}} & u_{x}^{3} d x \\
= & -\frac{1}{2} \int_{\mathbb{S}} u_{x}^{4} d x+\frac{3}{\alpha^{2}} \int_{\mathbb{S}} u_{x}^{2} u^{2} d x \\
& +\frac{3}{\alpha^{2}} \int_{\mathbb{S}} u_{x}^{2}\left(\frac{1}{2}(\rho+1)^{2}\right) d x \\
& -\frac{3}{\alpha^{2}} \int_{\mathbb{S}} u_{x}^{2} G *\left(u^{2}+\frac{\alpha^{2}}{2} u_{x}^{2}+\frac{1}{2}(\rho+1)^{2}\right) d x \\
\leq & -\frac{1}{2} \int_{\mathbb{S}} u_{x}^{4} d x+\frac{1}{4 \alpha^{2}}\left(\int_{\mathbb{S}} u_{x}^{2} d x\right)^{2} \\
& +\frac{3}{\alpha^{2}} \int_{\mathbb{S}} u_{x}^{2}\left(\frac{1}{2}(\rho+1)^{2}\right) d x \\
& -\frac{3}{2} \int_{\mathbb{S}} u_{x}^{2}\left(G * u_{x}^{2}\right) d x \\
\leq & -\frac{1}{2} \int_{\mathbb{S}} u_{x}^{4} d x+\frac{3}{\alpha^{2}} \int_{\mathbb{S}} u_{x}^{2}\left(\frac{1}{2}(\rho+1)^{2}\right) d x \\
& +\left(\frac{1}{4 \alpha^{2}}-\frac{3}{4 \alpha \sinh (1 / 2 \alpha)}\right)\left(\int_{\mathbb{S}} u_{x}^{2} d x\right)^{2} .
\end{aligned}
$$

Similar to Theorem 8 , assume that the solution $(u, \rho)$ of system (10) does not blow up; that is, there is $M_{1} \geq 0$, such that

$$
\inf _{(t, x) \in[0, T) \times \mathbb{R}} u_{x}(t, x) \geq-M_{1}
$$

then

$$
\|\rho(t, \cdot)\|_{L^{\infty}} \leq\left\|\rho_{0}\right\|_{L^{\infty}} \exp \left(M_{1} t\right) \doteq \widetilde{\kappa}
$$

So we can get

$$
\begin{aligned}
\frac{d}{d t} \int_{\mathbb{S}} u_{x}^{3} d x & \\
\leq & -\frac{1}{2} \int_{\mathbb{S}} u_{x}^{4} d x+\frac{3}{\alpha^{2}} \int_{\mathbb{S}} u_{x}^{2}\left(\frac{1}{2}(\rho+1)^{2}\right) d x \\
& +\left(\frac{1}{4 \alpha^{2}}-\frac{3}{4 \alpha \sinh (1 / 2 \alpha)}\right)\left(\int_{\mathbb{S}} u_{x}^{2} d x\right)^{2} \\
\leq & -\frac{1}{2} \int_{\mathbb{S}} u_{x}^{4} d x+\frac{3(\widetilde{\kappa}+1)^{2}}{2 \alpha^{2}} \int_{\mathbb{S}} u_{x}^{2} d x \\
& +\left(\frac{1}{4 \alpha^{2}}-\frac{3}{4 \alpha \sinh (1 / 2 \alpha)}\right)\left(\int_{\mathbb{S}} u_{x}^{2} d x\right)^{2}
\end{aligned}
$$

If $0<\sinh (1 / 2 \alpha) / \alpha<3$, then $\left(1 / 4 \alpha^{2}\right)-(3 / 4 \alpha \sinh (1 / 2 \alpha))<$ 0 . Since $\int_{\mathbb{S}}\left(u_{0}^{2}+\alpha^{2} u_{0 x}^{2}+\rho_{0}^{2}\right) d x>C_{0}$ for some constant $C_{0}$, due to Lemma 7 , there is some $\delta^{\star}>0$ such that

$$
\begin{aligned}
& \frac{3(\tilde{\kappa}+1)^{2}}{2 \alpha^{2}} \int_{\mathbb{S}} u_{x}^{2} d x \leq \delta^{\star}\left(\int_{\mathbb{S}} u_{x}^{2} d x\right)^{2}, \\
& \left(\frac{3}{4 \alpha \sinh (1 / 2 \alpha)}-\frac{1}{4 \alpha^{2}}-\delta^{\star}\right)>0 .
\end{aligned}
$$

Moreover, Lemma 7 also implies that

$$
\int_{\mathbb{S}} u_{x}^{2} d x \geq \frac{12}{12 \alpha^{2}+1}\|u(x)\|_{H_{\alpha}^{1}(\mathbb{S})}^{2} .
$$

Hence,

$$
\begin{aligned}
\frac{d}{d t} \int_{\mathbb{S}} & u_{x}^{3} d x \\
\leq & -\frac{1}{2} \int_{\mathbb{S}} u_{x}^{4} d x-\frac{144}{\left(12 \alpha^{2}+1\right)^{2}} \\
& \times\left(\frac{3}{4 \alpha \sinh (1 / 2 \alpha)}-\frac{1}{4 \alpha^{2}}-\delta^{\star}\right)\|u(x)\|_{H_{\alpha}^{1}(\mathbb{S})}^{4} .
\end{aligned}
$$

Note that $\|u(x)\|_{H_{\alpha}^{1}(\mathbb{S})}$ is bounded. In view of Hölder's inequality, there exists

$$
\int_{\mathbb{S}} u_{x}^{4} d x \geq\left(\int_{\mathbb{S}} u_{x}^{3} d x\right)^{4 / 3}
$$

For simplicity of notations, we denote by $\varphi(t)$ and $\mu$ the following quantities:

$$
\int_{\mathbb{S}} u_{x}^{3} d x, \frac{144}{\left(12 \alpha^{2}+1\right)^{2}}\left(\frac{3}{4 \alpha \sinh (1 / 2 \alpha)}-\frac{1}{4 \alpha^{2}}-\delta^{\star}\right)
$$

respectively. Therefore we have

$$
\frac{d \varphi(t)}{d t} \leq-\frac{1}{2} \varphi^{4 / 3}(t)-\mu\|u(x)\|_{H_{\alpha}^{1}(\mathbb{S})}^{4} .
$$

First, we can easily get $\varphi(t) \leq \varphi(0)-\mu\|u\|_{H_{\alpha}^{1}(\mathbb{S})}^{4} t$, and it is not difficult to find that there exists a time $t_{0}$ such that $\varphi\left(t_{0}\right)<0$. Then for all $t>t_{0}$, we have

$$
\frac{d \varphi(t)}{d t} \leq-\frac{1}{2} \varphi^{4 / 3}(t), \quad \text { with } \varphi\left(t_{0}\right)<0 .
$$

Solving this inequality yields

$$
\varphi(t) \leq\left(\varphi^{-1 / 3}\left(t_{0}\right)+\frac{1}{6}\left(t-t_{0}\right)\right)^{-3},
$$

which goes to $-\infty$ as $t$ tends to $-6 \varphi^{-1 / 3}\left(t_{0}\right)+t_{0}$; that is, there exists a time $T \leq-6 \varphi^{-1 / 3}\left(t_{0}\right)+t_{0}$ such that

$$
\lim _{t \uparrow T} \int_{\mathbb{S}} u_{x}^{3} d x=-\infty
$$


Since

$$
\int_{\mathbb{S}} u_{x}^{3} d x \geq \inf u_{x}(x, t) \int_{\mathbb{S}} u_{x}^{2} d x \geq c(\alpha) \inf u_{x}(x, t)\|u\|_{H_{\alpha}^{1}(\mathbb{S})}^{2},
$$

it shows that

$$
\lim _{t \uparrow T} \inf _{x \in \mathbb{R}} u_{x}(x, t)=-\infty
$$

Then it contradicts the assumption

$$
\inf _{(t, x) \in[0, T) \times \mathbb{R}} u_{x}(x, t)>-M_{1} .
$$

By Theorem 3, we know that the solution must blow up in finite time. This finishes the proof.

From now on, we give some blow-up criteria for the nonperiodic case.

Theorem 10. Assume that the initial data $\left(u_{0}, \rho_{0}\right) \in H^{s} \times$ $H^{s-1}, s \geq 2$, satisfy

$$
u_{0}^{\prime}\left(x_{0}\right)<-\sqrt{2 K_{0}}
$$

where $K_{0}$ is a constant determined later. And assume that $2 \omega+$ $\gamma / \alpha^{2}=0$ just for convenience. Then $T$ is finite and the slope of $u$ tends to negative infinity as $t$ goes to $T$ while $u$ is uniformly bounded on $[0, T)$.

Proof. Let $(u, \rho)$ be the solution to system (10) with the initial data $\left(u_{0}, \rho_{0}\right)$ and let $T$ be the maximal existence time of solution. Defining

$$
\begin{gathered}
m(t)=\inf _{x \in \mathbb{R}}\left[u_{x}(t, x)\right]=u_{x}(t, \xi(t)), \\
\gamma(t)=\rho(t, \xi(t)),
\end{gathered}
$$

one can get that $m$ is almost everywhere differentiable on $[0, T)$ with

$$
\frac{d m}{d t}(t)=u_{t x}(t, \xi(t))
$$

Note that $u_{x x}(t, \xi(t))=0$, and with (40), we have

$$
\begin{aligned}
m_{t}= & -m^{2}+\frac{1}{\alpha^{2}}\left(u^{2}+\frac{\alpha^{2}}{2} u_{x}^{2}+\frac{1}{2} \rho^{2}+\rho\right) \\
& -\frac{1}{\alpha^{2}} G *\left(u^{2}+\frac{\alpha^{2}}{2} u_{x}^{2}+\frac{1}{2} \rho^{2}+\rho\right),
\end{aligned}
$$

and with the relation $1 / 2=G *(1 / 2)$, we can get

$$
\begin{aligned}
m_{t}= & -m^{2}+\frac{1}{\alpha^{2}}\left(u^{2}+\frac{\alpha^{2}}{2} u_{x}^{2}+\frac{1}{2}(\rho+1)^{2}\right) \\
& -\frac{1}{\alpha^{2}} G *\left(u^{2}+\frac{\alpha^{2}}{2} u_{x}^{2}+\frac{1}{2}(\rho+1)^{2}\right) \\
\leq & -\frac{1}{2} m^{2}+\frac{1}{\alpha^{2}}\left(u^{2}+\frac{1}{2}(\rho+1)^{2}\right) .
\end{aligned}
$$

Since $u$ and $\rho$ are both bounded, then there exists a $K_{0}>0$, so that $\left(1 / \alpha^{2}\right)\left(u^{2}+(1 / 2)(\rho+1)^{2}\right)<K_{0}$, and thus we have

$$
m_{t} \leq-\frac{1}{2} m^{2}+K_{0}
$$

Note that if $m(0)<-\sqrt{2 K_{0}}$, then $m(t)<-\sqrt{2 K_{0}}$ for all $t \epsilon$ $[0, T)$. So there exists a $T$ such that

$$
\lim _{t \uparrow T} m(t)=-\infty
$$

This completes the proof of the theorem.

The authors in [25] claimed that the corresponding solution to the two-component Camassa-Holm equation exists globally in time provided that some initial data satisfy smallness conditions. However, we find surprisingly the smallness of initial energy can also lead to breaking wave solutions. This is stated as follows.

Theorem 11. Let $\left(u_{0}, \rho_{0}\right) \in H^{s}(\mathbb{R}) \times H^{s-1}(\mathbb{R})$ with $s>5 / 2$ and let $T>0$ be the maximal time of existence of the solution $(u, \rho)$ to (10) with initial data $\left(u_{0}, \rho_{0}\right)$. Assume there is some $x_{0} \in \mathbb{R}$ such that $\rho_{0}\left(x_{0}\right)=-1$, and there holds $0<\gamma<1-2 \omega \alpha^{2}$ for suitable $\alpha$ and $\gamma$. Moreover,

$$
\left\|\left(u_{0}, \rho_{0}\right)\right\|_{H_{\alpha}^{1} \times L^{2}}^{2}<\frac{\alpha^{5}\left(1-2 \omega \alpha^{2}-\gamma\right)}{2 \alpha^{4}+2 \omega \alpha^{2}+\gamma} .
$$

Then the corresponding solution to (10) blows up in finite time in the following sense, and there exists a $T_{0}$ with

$$
\begin{aligned}
0 & <T_{0} \\
& \leq 2+\frac{4 \alpha^{7}+4 \alpha^{7}\left|u_{0, x}\left(x_{0}\right)\right|}{\alpha^{5}-2 \omega \alpha^{7}-\gamma \alpha^{5}-\left(2 \alpha^{4}+2 \omega \alpha^{2}+\gamma\right)\left\|\left(u_{0}, \rho_{0}\right)\right\|_{H_{\alpha}^{1} \times L^{2}}^{2}},
\end{aligned}
$$

such that

$$
\lim _{t \uparrow T_{0}} \inf \left(\inf _{x \in \mathbb{R}} u_{x}(x, t)\right)=-\infty
$$

Proof. As in Theorem 8, we define

$$
M(t)=\inf _{x \in \mathbb{R}}\left[u_{x}(t, x)\right]=u_{x}(t, \xi(t)), \quad t \in[0, T),
$$

so that

$$
u_{x x}(t, \xi(t))=0, \quad \text { a.e. } t \in[0, T)
$$

We recall that $q(t, \cdot)$ defined by $(18)$ is a diffeomorphism of the line for any $t \in[0, T)$, so there exists an $x(t) \in \mathbb{R}$ such that

$$
q(t, x(t))=\xi(t), \quad t \in[0, T) .
$$


Differentiating the first equation of (10), we can get the expression of $M_{t}$ as follows:

$$
\begin{aligned}
& M_{t}(t, q(t, x(t))) \\
&=-M^{2}+\frac{1}{\alpha^{2}}\left(u^{2}+\frac{\alpha^{2}}{2} u_{x}^{2}+\frac{1}{2}(\rho+1)^{2}\right) \\
&-\frac{1}{\alpha^{2}} G *\left(u^{2}+\frac{\alpha^{2}}{2} u_{x}^{2}+\frac{1}{2}(\rho+1)^{2}\right) \\
&-\left(2 \omega+\frac{\gamma}{\alpha^{2}}\right) \partial_{x}^{2} G * u \\
&=-\frac{1}{2} M^{2}+\frac{1}{\alpha^{2}} u^{2}+\frac{1}{2 \alpha^{2}}(\rho+1)^{2} \\
&-\frac{1}{\alpha^{2}} G *\left(u^{2}+\frac{\alpha^{2}}{2} u_{x}^{2}\right)-\frac{1}{2 \alpha^{2}} G *(\rho+1)^{2} \\
&-\left(2 \omega+\frac{\gamma}{\alpha^{2}}\right) \partial_{x}^{2} G * u .
\end{aligned}
$$

Besides, along the trajectory of $q(t, x(t))$, we have

$$
\frac{d\left((\rho(t, q)+1) q_{x}\right)}{d t}=q_{x}\left(\rho_{t}+\rho_{x} u+\rho u_{x}+u_{x}\right)=0 .
$$

Then choose $\xi(0)=x_{0}$ and $\rho(\xi(0))=\rho_{0}\left(x_{0}\right)=-1$, which implies that

$$
\rho(t, \xi(t)) \equiv-1
$$

Therefore we can obtain

$$
\begin{aligned}
M_{t}= & -\frac{1}{2} M^{2}+\frac{1}{\alpha^{2}} u^{2}-\frac{1}{\alpha^{2}} G *\left(u^{2}+\frac{\alpha^{2}}{2} u_{x}^{2}\right) \\
& -\frac{1}{2 \alpha^{2}} G *(\rho+1)^{2}-\left(2 \omega+\frac{\gamma}{\alpha^{2}}\right) \partial_{x}^{2} G * u \\
\leq & -\frac{1}{2} M^{2}+\frac{1}{\alpha^{2}} u^{2}-\frac{1}{2 \alpha^{2}} u^{2}-\frac{1}{2 \alpha^{2}} G *(\rho+1)^{2} \\
& -\left(2 \omega+\frac{\gamma}{\alpha^{2}}\right) \partial_{x}^{2} G * u \\
= & -\frac{1}{2} M^{2}+\frac{1}{2 \alpha^{2}} u^{2}-\frac{1}{2 \alpha^{2}} G *(\rho+1)^{2} \\
& -\left(2 \omega+\frac{\gamma}{\alpha^{2}}\right) \partial_{x}^{2} G * u,
\end{aligned}
$$

where we use the fact

$$
G *\left(u^{2}+\frac{\alpha^{2}}{2} u_{x}^{2}\right) \geq \frac{1}{2} u^{2}
$$

We define

$$
f=\frac{1}{2 \alpha^{2}} u^{2}-\frac{1}{2 \alpha^{2}} G *(\rho+1)^{2}-\left(2 \omega+\frac{\gamma}{\alpha^{2}}\right) \partial_{x}^{2} G * u .
$$

For any $x \in \mathbb{R}$, then

$$
\begin{aligned}
u^{2}(x) & =\int_{-\infty}^{x_{0}} u u_{x} d x+\int_{\infty}^{x_{0}} u u_{x} d x \\
& \leq \frac{1}{2 \alpha}\left(\int_{-\infty}^{x_{0}}\left(u^{2}+\alpha^{2} u_{x}^{2}\right) d x+\int_{\infty}^{x_{0}}\left(u^{2}+\alpha^{2} u_{x}^{2}\right) d x\right) \\
& =\frac{1}{2 \alpha}\|u\|_{H_{\alpha}^{1}}^{2} \leq \frac{1}{2 \alpha}\|(u, \rho)\|_{H_{\alpha}^{1} \times L^{2}}^{2} .
\end{aligned}
$$

We also have

$$
\begin{gathered}
|G * \rho| \leq\|G\|_{L^{2}}\|\rho\|_{L^{2}}=\frac{1}{2 \sqrt{\alpha}}\|\rho\|_{L^{2}} \leq \frac{1}{4}+\frac{1}{4 \alpha}\|(u, \rho)\|_{H_{\alpha}^{1} \times L^{2}}^{2}, \\
\left|\partial_{x}^{2} G * u\right|=\left|G_{x} * u_{x}\right| \leq\left\|G_{x}\right\|_{L^{2}}\left\|u_{x}\right\|_{L^{2}} \\
=\frac{1}{2 \sqrt{\alpha^{3}}}\left\|u_{x}\right\|_{L^{2}} \leq \frac{1}{4}+\frac{1}{4 \alpha^{5}}\|(u, \rho)\|_{H_{\alpha}^{1} \times L^{2}}^{2} .
\end{gathered}
$$

Thus, we can get

$$
\begin{aligned}
f= & \frac{1}{2 \alpha^{2}} u^{2}-\frac{1}{2 \alpha^{2}} G * \rho^{2}-\frac{1}{\alpha^{2}} G * \rho \\
& -\frac{1}{2 \alpha^{2}}-\left(2 \omega+\frac{\gamma}{\alpha^{2}}\right) \partial_{x}^{2} G * u \\
\leq & \frac{1}{2 \alpha^{2}} u^{2}+\frac{1}{\alpha^{2}}|G * \rho|-\frac{1}{2 \alpha^{2}}+\left(2 \omega+\frac{\gamma}{\alpha^{2}}\right)\left|\partial_{x}^{2} G * u\right| \\
\leq & \frac{1}{4 \alpha^{3}}\|(u, \rho)\|_{H_{\alpha}^{1} \times L^{2}}^{2}+\frac{1}{4 \alpha^{3}}\|(u, \rho)\|_{H_{\alpha}^{1} \times L^{2}}^{2} \\
& +\frac{1}{4 \alpha^{5}}\left(2 \omega+\frac{\gamma}{\alpha^{2}}\right)\|(u, \rho)\|_{H_{\alpha}^{1} \times L^{2}}^{2}-\frac{1}{4 \alpha^{2}}+\frac{1}{4}\left(2 \omega+\frac{\gamma}{\alpha^{2}}\right) \\
\doteq & -a<0 .
\end{aligned}
$$

Therefore, when $\left\|\left(u_{0}, \rho_{0}\right)\right\|_{H^{1} \times L^{2}}^{2}<\left(\alpha^{5}-2 \omega \alpha^{7}-\gamma \alpha^{5}\right) /\left(2 \alpha^{4}+\right.$ $\left.2 \omega \alpha^{2}+\gamma\right)$ holds, we always have $f<0$. In this case, we can obtain

$$
M^{\prime}(t) \leq-\frac{1}{2} M^{2}-a<0, \quad t \in[t, T)
$$

so $M(t)$ is decreasing strictly in such interval. Assume that the solution $(u, \rho)$ of $(10)$ does not blow up; that is, it exists globally in time; that is, $T=\infty$. Then integrating (92) over $\left[0, t_{1}\right]$ with $t_{1}=\left(1+\left|u_{0, x}\left(x_{0}\right)\right|\right) / a$ yields

$$
M\left(t_{1}\right)=M(0)-\int_{0}^{t_{1}} M^{\prime}(t) d t \leq\left|u_{0, x}\left(x_{0}\right)\right|-a t_{1} \leq-1 .
$$

We can also find that $M^{\prime}(t) \leq-(1 / 2) M^{2}$, which leads to

$$
-\frac{d}{d t}\left(\frac{1}{M(t)}\right)=\frac{1}{M^{2}(t)} \frac{d}{d t} M(t) \leq-\frac{1}{2}, \quad t \in\left[t_{1}, T\right) .
$$


Integrating both sides, we have

$$
-\frac{1}{M(t)}-1 \leq-\frac{1}{M(t)}+\frac{1}{M\left(t_{1}\right)} \leq-\frac{1}{2}\left(t-t_{1}\right),
$$

where we use the result $M\left(t_{1}\right) \leq-1$ obtained previously. Solve the inequality

$$
M(t) \leq \frac{2}{\left(t-t_{1}\right)-2} \longrightarrow-\infty, \quad \text { as } t \longrightarrow t_{1}+2 .
$$

That implies that $T \leq t_{1}+2<\infty$, which leads to a contradiction as $t \rightarrow \infty$. This completes the proof.

\section{Persistence Properties}

Attention now is turned to determining the persistence properties of solutions to the two-component DGH equation. We show that certain decay properties of the initial data persist in corresponding solution in later time as long as it exists. Precisely, we prove that the corresponding solution $(u, \rho)$ and its first order spatial derivatives preserve the exponential decay as their initial values do. This is a very important investigation since we can obtain the detailed asymptotic behavior of solution from the initial values. The main idea comes from the recent works of Zhou et al. [17, 38]. Now, we state our result as follows.

Theorem 12. Assume that for some $T>0$ and $s>5 / 2$, $(u, \rho) \in C\left([0, T] ; H^{s} \times H^{s-1}\right)$ is a strong solution of the initial value problem to the system (10) and $2 \omega+\gamma / \alpha^{2}=0$ just for convenience. If $u_{0}=u(x, 0), \rho_{0}=\rho(x, 0)$ satisfy

$$
\begin{array}{r}
\left|u_{0}(x)\right|,\left|\partial_{x} u_{0}(x)\right|,\left|\rho_{0}(x)\right|,\left|\partial_{x} \rho_{0}(x)\right| \sim O\left(e^{-\theta x}\right) \\
\text { as } x \longrightarrow \infty
\end{array}
$$

for some $\theta \in(0,1)$. Then

$$
\begin{array}{r}
|u(x, t)|,\left|u_{x}(x, t)\right|,|\rho(x, t)|,\left|\rho_{x}(x, t)\right| \sim O\left(e^{-\theta x}\right) \\
\text { as } x \longrightarrow \infty
\end{array}
$$

uniformly in the time interval $[0, T]$.

\section{Notation. Consider}

$$
\begin{aligned}
& u(x, t) \sim O\left(e^{-\theta x}\right), \quad \text { as } x \longrightarrow \infty, \text { if } \lim _{x \rightarrow \infty} \frac{|u(x)|}{e^{-\theta x}}=L, \\
& u(x, t) \sim \mathrm{o}\left(e^{-\theta x}\right), \quad \text { as } x \longrightarrow \infty, \text { if } \lim _{x \rightarrow \infty} \frac{|u(x)|}{e^{-\theta x}}=0 .
\end{aligned}
$$

Proof. First, we figure out the estimates on $\|u(x, t)\|_{L^{\infty}}$, $\left\|u_{x}(x, t)\right\|_{L^{\infty}},\|\rho(x, t)\|_{L^{\infty}}$, and $\left\|\rho_{x}(x, t)\right\|_{L^{\infty}}$. Then we use the weight function to obtain the desired result.

Step 1. Estimate for $\|u(x, t)\|_{L^{\infty}},\|\rho(x, t)\|_{L^{\infty}}$.
Multiplying the first equation of (10) by $u^{2 n-1}$ with $n \in \mathbb{Z}$ and then integrating both sides with respect to $x$-variable, we can get

$$
\begin{aligned}
& \int_{\mathbb{R}} u^{2 n-1} u_{t} d x \\
& \quad+\int_{\mathbb{R}} u^{2 n-1} u u_{x} d x+\int_{\mathbb{R}} u^{2 n-1} 2 \omega u_{x} d x \\
& \quad+\int_{\mathbb{R}} u^{2 n-1} \partial_{x} p *\left(u^{2}+\frac{\alpha^{2}}{2} u_{x}^{2}+\frac{1}{2} \rho^{2}+\rho\right) d x=0 .
\end{aligned}
$$

The first term of the previous identity is

$$
\int_{\mathbb{R}} u^{2 n-1} u_{t} d x=\frac{1}{2 n} \frac{d}{d t}\|u(t)\|_{L^{2 n}}^{2 n}=\|u(t)\|_{L^{2 n}}^{2 n-1} \frac{d}{d t}\|u(t)\|_{L^{2 n}}
$$

and the estimate of the second term is

$$
\left|\int_{\mathbb{R}} u^{2 n-1} u u_{x} d x\right| \leq\left\|u_{x}(t)\right\|_{L^{\infty}}\|u(t)\|_{L^{2 n}}^{2 n} .
$$

In view of Hölder's inequality, we can obtain the following estimate for the third and the last terms in (100):

$$
\begin{gathered}
\int_{\mathbb{R}} 2 \omega u^{2 n-1} u_{x} d x \leq 2 \omega\|u(t)\|_{L^{2 n}}^{2 n-1}\left\|u_{x}(t)\right\|_{L^{2 n}}, \\
\int_{\mathbb{R}} u^{2 n-1} \partial_{x} p *\left(u^{2}+\frac{\alpha^{2}}{2} u_{x}^{2}+\frac{1}{2} \rho^{2}+\rho\right) d x \\
\leq\|u(t)\|_{L^{2 n}}^{2 n-1}\left\|\partial_{x} p *\left(u^{2}+\frac{\alpha^{2}}{2} u_{x}^{2}+\frac{1}{2} \rho^{2}+\rho\right)\right\|_{L^{2 n}} .
\end{gathered}
$$

Putting all the previous inequalities into (100) yields

$$
\begin{aligned}
\frac{d}{d t}\|u(t)\|_{L^{2 n}} \leq & \left\|u_{x}(t)\right\|_{L^{\infty}}\|u(t)\|_{L^{2 n}}+2 \omega\left\|u_{x}(t)\right\|_{L^{2 n}} \\
& +\left\|\partial_{x} p *\left(u^{2}+\frac{\alpha^{2}}{2} u_{x}^{2}+\frac{1}{2} \rho^{2}+\rho\right)\right\|_{L^{2 n}} .
\end{aligned}
$$

Thus, according to the Sobolev embedding theorem, there exists a constant

$$
M=\sup _{t \in[0, T]}\|u(x, t)\|_{H^{s}}
$$

such that applying Gronwall's inequality gives us

$$
\begin{aligned}
& \|u(t)\|_{L^{2 n}} \\
& \leq\left(\|u(0)\|_{L^{2 n}}+\int_{0}^{t} 2 \omega\left\|u_{x}(\tau)\right\|_{L^{2 n}}\right. \\
& \left.\quad+\int_{0}^{t}\left\|\partial_{x} p *\left(u^{2}+\frac{\alpha^{2}}{2} u_{x}^{2}+\frac{1}{2} \rho^{2}+\rho\right)\right\|_{L^{2 n}} d \tau\right) e^{M t} .
\end{aligned}
$$


For any $f \in L^{1}(\mathbb{R}) \cap L^{\infty}(\mathbb{R})$, we know that

$$
\lim _{q \rightarrow \infty}\|f\|_{L^{q}}=\|f\|_{L^{\infty}} .
$$

Thus, taking the limits in (106), we get

$\|u(t)\|_{L^{\infty}}$

$$
\begin{aligned}
& \leq\left(\|u(0)\|_{L^{\infty}}+\int_{0}^{t} 2 \omega\left\|u_{x}(\tau)\right\|_{L^{\infty}} d \tau\right. \\
& \left.\quad+\int_{0}^{t}\left\|\partial_{x} p *\left(u^{2}+\frac{\alpha^{2}}{2} u_{x}^{2}+\frac{1}{2} \rho^{2}+\rho\right)\right\|_{L^{\infty}} d \tau\right) e^{M t} .
\end{aligned}
$$

Similarly, for the second equation of (10), we have

$$
\begin{aligned}
& \int_{\mathbb{R}} \rho^{2 n-1} \rho_{t} d x+\int_{\mathbb{R}} \rho^{2 n-1} \rho_{x} u d x \\
& \quad+\int_{\mathbb{R}} \rho^{2 n-1} \rho u_{x} d x+\int_{\mathbb{R}} \rho^{2 n-1} u_{x} d x=0,
\end{aligned}
$$

and using the same method, one can get

$$
\frac{d}{d t}\|\rho(t)\|_{L^{2 n}} \leq\left\|u_{x}\right\|_{L^{\infty}}\|\rho(t)\|_{L^{2 n}}+\|u\|_{L^{\infty}}\left\|\rho_{x}\right\|_{L^{2 n}}+\left\|u_{x}\right\|_{L^{2 n}}
$$

Using the Gronwall's inequality, one gets

$$
\begin{aligned}
& \|\rho(t)\|_{L^{2 n}} \\
& \quad \leq\left(\|\rho(0)\|_{L^{2 n}}+\int_{0}^{t}\left(\|u\|_{L^{\infty}}\left\|\rho_{x}\right\|_{L^{2 n}}+\left\|u_{x}\right\|_{L^{2 n}}\right) d \tau\right) e^{M t} .
\end{aligned}
$$

Taking the limits, one gets

$$
\begin{aligned}
& \|\rho(t)\|_{L^{\infty}} \\
& \leq\left(\|\rho(0)\|_{L^{\infty}}+\int_{0}^{t}\left(\|u\|_{L^{\infty}}\left\|\rho_{x}\right\|_{L^{\infty}}+\left\|u_{x}\right\|_{L^{\infty}}\right) d \tau\right) e^{M t} .
\end{aligned}
$$

Step 2. Estimate for $\left\|u_{x}(x, t)\right\|_{L^{\infty}},\left\|\rho_{x}(x, t)\right\|_{L^{\infty}}$.

We will establish an estimate on $\left\|u_{x}(x, t)\right\|_{L^{\infty}}$ using the same method. Differentiating the first equation (10) with respect to $x$-variable produces the following equation:

$$
\begin{aligned}
u_{x t}+ & u u_{x x}+u_{x}^{2}+2 \omega u_{x x} \\
& +\partial_{x}^{2} p *\left(u^{2}+\frac{\alpha^{2}}{2} u_{x}^{2}+\frac{1}{2} \rho^{2}+\rho\right)=0 .
\end{aligned}
$$

Then, multiplying (113) by $u_{x}^{2 n-1}$ with $n \in \mathbb{Z}^{+}$, integrating the result in the $x$-variable, and considering the second term in the previous identity with integration by parts, one can get

$$
\int_{\mathbb{R}} u u_{x x} u_{x}^{2 n-1} d x=\int_{\mathbb{R}} u\left(\frac{u_{x}^{2 n}}{2 n}\right)_{x} d x=-\frac{1}{2 n} \int_{\mathbb{R}} u_{x} u_{x}^{2 n} d x,
$$

so we have

$$
\begin{aligned}
& \int_{\mathbb{R}} u_{x}^{2 n-1} u_{x t} d x-\frac{1}{2 n} \int_{\mathbb{R}} u_{x} u_{x}^{2 n} d x+\int_{\mathbb{R}} u_{x}^{2 n-1} u_{x}^{2} d x \\
& \quad+\int_{\mathbb{R}} u_{x}^{2 n-1} 2 \omega u_{x x} d x \\
& \quad+\int_{\mathbb{R}} u_{x}^{2 n-1} \partial_{x}^{2} p *\left(u^{2}+\frac{\alpha^{2}}{2} u_{x}^{2}+\frac{1}{2} \rho^{2}+\rho\right) d x=0 .
\end{aligned}
$$

Similarly, we can get the following inequality:

$$
\begin{aligned}
\frac{d}{d t}\left\|u_{x}(t)\right\|_{L^{2 n}} \leq & 2\left\|u_{x}(t)\right\|_{L^{\infty}}\left\|u_{x}(t)\right\|_{L^{2 n}}+2 \omega\left\|u_{x x}(t)\right\|_{L^{2 n}} \\
& +\left\|\partial_{x}^{2} p *\left(u^{2}+\frac{\alpha^{2}}{2} u_{x}^{2}+\frac{1}{2} \rho^{2}+\rho\right)\right\|_{L^{2 n}}
\end{aligned}
$$

Using Gronwall's inequality, we can similarly get

$$
\begin{aligned}
& \left\|u_{x}(t)\right\|_{L^{2 n}} \\
& \leq e^{2 M t}\left(\left\|u_{x}(0)\right\|_{L^{2 n}}+\int_{0}^{t} 2 \omega\left\|u_{x x}(\tau)\right\|_{L^{2 n}} d \tau\right. \\
& \left.\quad+\int_{0}^{t}\left\|\partial_{x}^{2} p *\left(u^{2}+\frac{\alpha^{2}}{2} u_{x}^{2}+\frac{1}{2} \rho^{2}+\rho\right)\right\|_{L^{2 n}} d \tau\right) .
\end{aligned}
$$

Taking the limits, one gets

$$
\begin{aligned}
& \left\|u_{x}(t)\right\|_{L^{\infty}} \\
& \leq e^{2 M t}\left(\left\|u_{x}(0)\right\|_{L^{\infty}}+\int_{0}^{t} 2 \omega\left\|u_{x x}(\tau)\right\|_{L^{\infty}} d \tau\right. \\
& \left.\quad+\int_{0}^{t}\left\|\partial_{x}^{2} p *\left(u^{2}+\frac{\alpha^{2}}{2} u_{x}^{2}+\frac{1}{2} \rho^{2}+\rho\right)\right\|_{L^{\infty}} d \tau\right) .
\end{aligned}
$$

Differentiating the second equation of (10), one has

$$
\rho_{t x}+\rho_{x x} u+2 \rho_{x} u_{x}+\rho u_{x x}+u_{x x}=0
$$

and using the same method, we can obtain

$$
\begin{gathered}
\int_{\mathbb{R}} \rho_{x}^{2 n-1} \rho_{t x} d x+\int_{\mathbb{R}} \rho_{x}^{2 n-1} \rho_{x x} u d x+2 \int_{\mathbb{R}} \rho_{x}^{2 n-1} \rho_{x} u_{x} d x \\
+\int_{\mathbb{R}} \rho_{x}^{2 n-1} \rho u_{x x} d x+\int_{\mathbb{R}} \rho_{x}^{2 n-1} u_{x x} d x=0 .
\end{gathered}
$$

In a similar way, we should deal with the second term as follows:

$$
\left|\int_{\mathbb{R}} \rho_{x}^{2 n-1} \rho_{x x} u d x\right|=\left|\int_{\mathbb{R}}\left(\frac{\rho_{x}^{2 n}}{2 n}\right)_{x} u d x\right|=\left|\frac{1}{2 n} \int_{\mathbb{R}} \rho_{x}^{2 n} u_{x} d x\right|,
$$


thus we can get the estimates of $\left\|\rho_{x}(x, t)\right\|_{L^{\infty}}$ as follows:

$$
\begin{aligned}
& \left\|\rho_{x}(t)\right\|_{L^{\infty}} \\
& \quad \leq e^{3 M t}\left(\left\|\rho_{x}(0)\right\|_{L^{\infty}}+\int_{0}^{t}\left(\left\|u_{x x}\right\|_{L^{\infty}}\|\rho\|_{L^{\infty}}+\left\|u_{x x}\right\|_{L^{\infty}}\right) d \tau\right) .
\end{aligned}
$$

In what follows, we use the weight function to get the desired result. We shall now introduce the weight function $\varphi_{N}(x)$ with $N \in \mathbb{Z}^{+}$, which is independent of $t$ as follows:

$$
\varphi_{N}(x)= \begin{cases}1, & x \leq 0 \\ e^{\theta x}, & x \in(0, N) \\ e^{\theta N}, & x \geq N\end{cases}
$$

Note that $0 \leq \varphi_{N}^{\prime}(x) \leq \varphi_{N}(x)$. Multiplying the first equation of (10) and (106) by $\varphi_{N}$, we have

$$
\begin{aligned}
\varphi_{N} u_{t} & +\varphi_{N} u u_{x}+2 \omega \varphi_{N} u_{x} \\
& +\varphi_{N} \partial_{x} p *\left(u^{2}+\frac{\alpha^{2}}{2} u_{x}^{2}+\frac{1}{2} \rho^{2}+\rho\right)=0, \\
\varphi_{N} u_{x t} & +\varphi_{N} u u_{x x}+\varphi_{N} u_{x}^{2}+2 \omega \varphi_{N} u_{x x} \\
& +\varphi_{N} \partial_{x}^{2} p *\left(u^{2}+\frac{\alpha^{2}}{2} u_{x}^{2}+\frac{1}{2} \rho^{2}+\rho\right)=0 .
\end{aligned}
$$

In order to get the estimate of $u_{x} \varphi_{N}$, we need to eliminate the second derivatives as follows:

$$
\begin{aligned}
& \left|\int_{\mathbb{R}} \varphi_{N} u u_{x x}\left(u_{x} \varphi_{N}\right)^{2 n-1} d x\right| \\
& \quad=\left|\int_{\mathbb{R}} u\left(u_{x} \varphi_{N}\right)^{2 n-1}\left(\left(u_{x} \varphi_{N}\right)_{x}-u_{x} \varphi_{N}^{\prime}\right) d x\right| \\
& \quad=\left|\int_{\mathbb{R}} u\left(\frac{\left(u_{x} \varphi_{N}\right)^{2 n}}{2 n}\right)_{x} d x-\int_{\mathbb{R}} u u_{x} \varphi_{N}^{\prime}\left(u_{x} \varphi_{N}\right)^{2 n-1} d x\right| \\
& \quad \leq 2\left(\|u(t)\|_{L^{\infty}}+\left\|u_{x}(t)\right\|_{L^{\infty}}\right)\left\|u_{x} \varphi_{N}\right\|_{L^{2 n}}^{2 n},
\end{aligned}
$$

where we use the fact $0 \leq \varphi_{N}^{\prime}(x) \leq \varphi_{N}(x)$. Hence, as in the weightless case, we get the following inequality in view of the estimates of $\|u(x, t)\|_{L^{\infty}}$ and $\left\|u_{x}(x, t)\right\|_{L^{\infty}}$ :

$$
\begin{aligned}
& \left\|u(t) \varphi_{N}\right\|_{L^{\infty}}+\left\|u_{x}(t) \varphi_{N}\right\|_{L^{\infty}} \\
& \leq e^{2 M t}\left(\left\|u(0) \varphi_{N}\right\|_{L^{\infty}}+\left\|u_{x}(0) \varphi_{N}\right\|_{L^{\infty}}\right) \\
& \quad+e^{2 M t} \int_{0}^{t}\left\|\varphi_{N} \partial_{x} p *\left(u^{2}+\frac{\alpha^{2}}{2} u_{x}^{2}+\frac{1}{2} \rho^{2}+\rho\right)\right\|_{L^{\infty}} d \tau \\
& \quad+e^{2 M t} \int_{0}^{t}\left\|\varphi_{N} \partial_{x}^{2} p *\left(u^{2}+\frac{\alpha^{2}}{2} u_{x}^{2}+\frac{1}{2} \rho^{2}+\rho\right)\right\|_{L^{\infty}} d \tau .
\end{aligned}
$$

Similarly, we get estimates for $\rho \varphi_{N}$ and $\rho_{x} \varphi_{N}$ as follows:

$$
\begin{aligned}
& \left\|\rho \varphi_{N}\right\|_{L^{\infty}}+\left\|\rho_{x} \varphi_{N}\right\|_{L^{\infty}} \\
& \quad \leq e^{2 M t}\left(\left\|\rho(0) \varphi_{N}\right\|_{L^{\infty}}+\left\|\rho_{x}(0) \varphi_{N}\right\|_{L^{\infty}}\right) .
\end{aligned}
$$

On the other hand, a simple calculation shows that there exists $C>0$, depending only on $\theta \in(0,1)$ such that for any $N \in \mathbb{Z}^{+}$,

$$
\varphi_{N}(x) \int_{\mathbb{R}} e^{-|(x-y) / \alpha|} \frac{1}{\varphi_{N}(y)} d y \leq C .
$$

Therefore for any appropriate function $g$, one can get

$$
\begin{aligned}
& \left|\varphi_{N} \partial_{x} G(x) * g^{2}(x)\right| \\
& \quad=\left|\frac{1}{2 \alpha} \varphi_{N}(x) \int_{\mathbb{R}} e^{-|(x-y) / \alpha|} g^{2}(y) d y\right| \\
& \quad=\frac{1}{2 \alpha} \varphi_{N}(x) \int_{\mathbb{R}} e^{-|(x-y) / \alpha|} \frac{1}{\varphi_{N}(y)} \varphi_{N}(y) g(y) g(y) d y \\
& \quad \leq \frac{1}{2 \alpha}\left(\varphi_{N}(x) \int_{\mathbb{R}} e^{-|(x-y) / \alpha|} \frac{1}{\varphi_{N}(y)} d y\right)\left\|g \varphi_{N}\right\|_{L^{\infty}}\|g\|_{L^{\infty}} \\
& \quad \leq C^{\prime}\left\|g \varphi_{N}\right\|_{L^{\infty}}\|g\|_{L^{\infty}},
\end{aligned}
$$

and using the same method,

$$
\left|\varphi_{N} \partial_{x}^{2} G(x) * g^{2}(x)\right| \leq C^{\prime}\left\|g \varphi_{N}\right\|_{L^{\infty}}\|g\|_{L^{\infty}} .
$$

Besides,

$$
\begin{aligned}
& \left|\varphi_{N} \partial_{x}^{2} p * \rho\right| \\
& \quad=\left|\varphi_{N} p_{x} * \rho_{x}\right| \leq\left|\varphi_{N}\right|\left\|p_{x}\right\|_{L^{2}}\left\|\rho_{x}\right\|_{L^{2}} \leq C^{\prime}\left\|\varphi_{N} \rho_{x}\right\|_{L^{\infty}} .
\end{aligned}
$$

In a similar way, we have

$$
\left|\varphi_{N} \partial_{x} p * \rho\right| \leq C^{\prime}\left\|\varphi_{N} \rho\right\|_{L^{\infty}} .
$$

Then we can get the following estimate:

$$
\begin{gathered}
\left\|u(t) \varphi_{N}\right\|_{L^{\infty}}+\left\|u_{x}(t) \varphi_{N}\right\|_{L^{\infty}}+\left\|\rho(t) \varphi_{N}\right\|_{L^{\infty}}+\left\|\rho_{x}(t) \varphi_{N}\right\|_{L^{\infty}} \\
\leq \kappa_{0}\left(\left\|u(0) \varphi_{N}\right\|_{L^{\infty}}+\left\|u_{x}(0) \varphi_{N}\right\|_{L^{\infty}}\right. \\
\left.+\left\|\rho(0) \varphi_{N}\right\|_{L^{\infty}}+\left\|\rho_{x}(0) \varphi_{N}\right\|_{L^{\infty}}\right) \\
+\kappa_{0}\left(\int _ { 0 } ^ { t } \left(\left\|u(t) \varphi_{N}\right\|_{L^{\infty}}+\left\|u_{x}(t) \varphi_{N}\right\|_{L^{\infty}}\right.\right. \\
\left.\left.+\left\|\rho(t) \varphi_{N}\right\|_{L^{\infty}}+\left\|\rho_{x}(t) \varphi_{N}\right\|_{L^{\infty}}\right) d t\right) .
\end{gathered}
$$

Using Gronwall's inequality of integral form, one can get

$$
\begin{gathered}
\left\|u(t) \varphi_{N}\right\|_{L^{\infty}}+\left\|u_{x}(t) \varphi_{N}\right\|_{L^{\infty}}+\left\|\rho(t) \varphi_{N}\right\|_{L^{\infty}}+\left\|\rho_{x}(t) \varphi_{N}\right\|_{L^{\infty}} \\
\leq \kappa_{0}\left(\left\|u(0) \varphi_{N}\right\|_{L^{\infty}}+\left\|u_{x}(0) \varphi_{N}\right\|_{L^{\infty}}\right. \\
\left.+\left\|\rho(0) \varphi_{N}\right\|_{L^{\infty}}+\left\|\rho_{x}(0) \varphi_{N}\right\|_{L^{\infty}}\right) .
\end{gathered}
$$


Taking the limit as $N$ goes to infinity, we can obtain

$$
\begin{gathered}
\left|u(x, t) e^{\theta x}\right|+\left|u_{x}(x, t) e^{\theta x}\right|+\left|\rho(x, t) e^{\theta x}\right|+\left|\rho_{x}(x, t) e^{\theta x}\right| \\
\leq \kappa_{0}\left(\left\|u(0) e^{\theta x}\right\|+\left\|u_{x}(0) e^{\theta x}\right\|\right. \\
\left.+\left\|\rho(0) e^{\theta x}\right\|+\left\|\rho_{x}(0) e^{\theta x}\right\|\right) .
\end{gathered}
$$

This completes the proof.

\section{Acknowledgments}

This work was partially supported by Zhejiang Provincial Natural Science Foundation of China under LQ12A01009 and LY12A01014 and NSFC under Grant no. 11226172.

\section{References}

[1] H. R. Dullin, G. A. Gottwald, and D. D. Holm, "An integrable shallow water equation with linear and nonlinear dispersion," Physical Review Letters, vol. 87, no. 19, Article ID 194501, 4 pages, 2001.

[2] J. Bourgain, "Fourier transform restriction phenomena for certain lattice subsets and applications to nonlinear evolution equations," Geometric and Functional Analysis, vol. 3, no. 3, pp. 209-262, 1993.

[3] C. E. Kenig, G. Ponce, and L. Vega, "Well-posedness and scattering results for the generalized Korteweg-de Vries equation via the contraction principle," Communications on Pure and Applied Mathematics, vol. 46, no. 4, pp. 527-620, 1993.

[4] P. G. Drazin and R. S. Johnson, Solitons: An Introduction, Cambridge University Press, Cambridge, Mass, USA, 1989.

[5] R. Camassa and D. D. Holm, "An integrable shallow water equation with peaked solitons," Physical Review Letters, vol. 71, no. 11, pp. 1661-1664, 1993.

[6] A. A. Himonas and G. Misiołek, "The Cauchy problem for an integrable shallow-water equation," Differential and Integral Equations, vol. 14, no. 7, pp. 821-831, 2001.

[7] Y. A. Li and P. J. Olver, "Well-posedness and blow-up solutions for an integrable nonlinearly dispersive model wave equation," Journal of Differential Equations, vol. 162, no. 1, pp. 27-63, 2000.

[8] G. Misiołek, "Classical solutions of the periodic Camassa-Holm equation," Geometric and Functional Analysis, vol. 12, no. 5, pp. 1080-1104, 2002.

[9] S. Shkoller, "Geometry and curvature of diffeomorphism groups with $H^{1}$ metric and mean hydrodynamics," Journal of Functional Analysis, vol. 160, no. 1, pp. 337-365, 1998.

[10] A. Constantin and J. Escher, "Wave breaking for nonlinear nonlocal shallow water equations," Acta Mathematica, vol. 181, no. 2, pp. 229-243, 1998.

[11] A. Constantin and J. Escher, "Well-posedness, global existence, and blowup phenomena for a periodic quasi-linear hyperbolic equation," Communications on Pure and Applied Mathematics, vol. 51, no. 5, pp. 475-504, 1998.

[12] Z. Jiang, L. Ni, and Y. Zhou, "Wave breaking of the CamassaHolm equation," Journal of Nonlinear Science, vol. 22, no. 2, pp. 235-245, 2012.

[13] H. P. McKean, "Breakdown of a shallow water equation," The Asian Journal of Mathematics, vol. 2, no. 4, pp. 867-874, 1998.
[14] Y. Zhou, "Wave breaking for a periodic shallow water equation," Journal of Mathematical Analysis and Applications, vol. 290, no. 2, pp. 591-604, 2004.

[15] Y. Zhou, "Wave breaking for a shallow water equation," Nonlinear Analysis: Theory, Methods \& Applications, vol. 57, no. 1, pp. 137-152, 2004.

[16] Z. Xin and P. Zhang, "On the weak solutions to a shallow water equation," Communications on Pure and Applied Mathematics, vol. 53, no. 11, pp. 1411-1433, 2000.

[17] A. A. Himonas, G. Misiołek, G. Ponce, and Y. Zhou, "Persistence properties and unique continuation of solutions of the Camassa-Holm equation," Communications in Mathematical Physics, vol. 271, no. 2, pp. 511-522, 2007.

[18] F. Cooper and H. Shepard, "Solitons in the Camassa-Holm shallow water equation," Physics Letters A, vol. 194, no. 4, pp. 246-250, 1994.

[19] L. Tian and X. Song, "New peaked solitary wave solutions of the generalized Camassa-Holm equation," Chaos, Solitons and Fractals, vol. 19, no. 3, pp. 621-637, 2004.

[20] L. Tian and J. Yin, "New compacton solutions and solitary wave solutions of fully nonlinear generalized Camassa-Holm equations," Chaos, Solitons and Fractals, vol. 20, no. 2, pp. 289299, 2004.

[21] L. Tian, G. Gui, and Y. Liu, "On the well-posedness problem and the scattering problem for the Dullin-Gottwald-Holm equation," Communications in Mathematical Physics, vol. 257, no. 3, pp. 667-701, 2005.

[22] Y. Liu, "Global existence and blow-up solutions for a nonlinear shallow water equation," Mathematische Annalen, vol. 335, no. 3, pp. 717-735, 2006.

[23] Y. Zhou, "Blow-up of solutions to the DGH equation," Journal of Functional Analysis, vol. 250, no. 1, pp. 227-248, 2007.

[24] Y. Zhou and Z. Guo, "Blow up and propagation speed of solutions to the DGH equation," Discrete and Continuous Dynamical Systems B, vol. 12, no. 3, pp. 657-670, 2009.

[25] A. Constantin and R. I. Ivanov, "On an integrable twocomponent Camassa-Holm shallow water system," Physics Letters A, vol. 372, no. 48, pp. 7129-7132, 2008.

[26] J. Escher, O. Lechtenfeld, and Z. Yin, "Well-posedness and blowup phenomena for the 2-component Camassa-Holm equation," Discrete and Continuous Dynamical Systems A, vol. 19, no. 3, pp. 493-513, 2007.

[27] Y. Fu, Y. Liu, and C. Qu, "Well-posedness and blow-up solution for a modified two-component periodic Camassa-Holm system with peakons," Mathematische Annalen, vol. 348, no. 2, pp. 415448, 2010.

[28] C. Guan and Z. Yin, "Global existence and blow-up phenomena for an integrable two-component Camassa-Holm shallow water system," Journal of Differential Equations, vol. 248, no. 8, pp. 2003-2014, 2010.

[29] G. Gui and Y. Liu, "On the global existence and wave-breaking criteria for the two-component Camassa-Holm system," Journal of Functional Analysis, vol. 258, no. 12, pp. 4251-4278, 2010.

[30] Z. Guo, "Blow-up and global solutions to a new integrable model with two components," Journal of Mathematical Analysis and Applications, vol. 372, no. 1, pp. 316-327, 2010.

[31] Z. Guo and Y. Zhou, "On solutions to a two-component generalized Camassa-Holm equation," Studies in Applied Mathematics, vol. 124, no. 3, pp. 307-322, 2010.

[32] Z. Guo and M. Zhu, "Wave breaking for a modified twocomponent Camassa-Holm system," Journal of Differential Equations, vol. 252, no. 3, pp. 2759-2770, 2012. 
[33] F. Guo, H. Gao, and Y. Liu, "On the wave-breaking phenomena for the two-component Dullin-Gottwald-Holm system," Journal of the London Mathematical Society, vol. 86, no. 3, pp. 810834, 2012.

[34] M. Zhu and J. Xu, "On the wave-breaking phenomena for the periodic two-component Dullin-Gottwald-Holm system," Journal of Mathematical Analysis and Applications, vol. 391, no. 2, pp. 415-428, 2012.

[35] T. Kato, "On the Cauchy problem for the (generalized) Korteweg-de Vries equation," in Studies in Applied Mathematics, vol. 8, pp. 93-128, Academic Press, New York, NY, USA, 1983.

[36] P. Zhang and Y. Liu, "Stability of solitary waves and wavebreaking phenomena for the two-component Camassa-Holm system," International Mathematics Research Notices, vol. 2010, no. 11, pp. 1981-2021, 2010.

[37] Y. Zhou, "Blow-up of solutions to a nonlinear dispersive rod equation," Calculus of Variations and Partial Differential Equations, vol. 25, no. 1, pp. 63-77, 2006.

[38] Z. Guo and Y. Zhou, "Wave breaking and persistence properties for the dispersive rod equation," SIAM Journal on Mathematical Analysis, vol. 40, no. 6, pp. 2567-2580, 2009. 


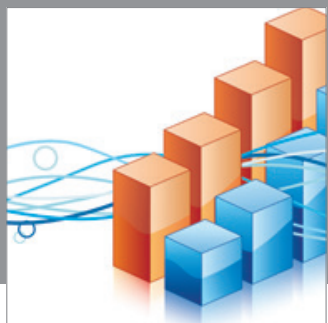

Advances in

Operations Research

mansans

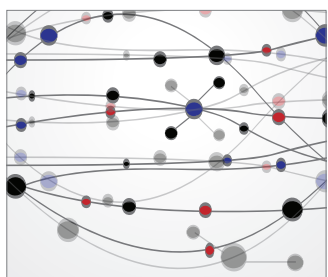

The Scientific World Journal
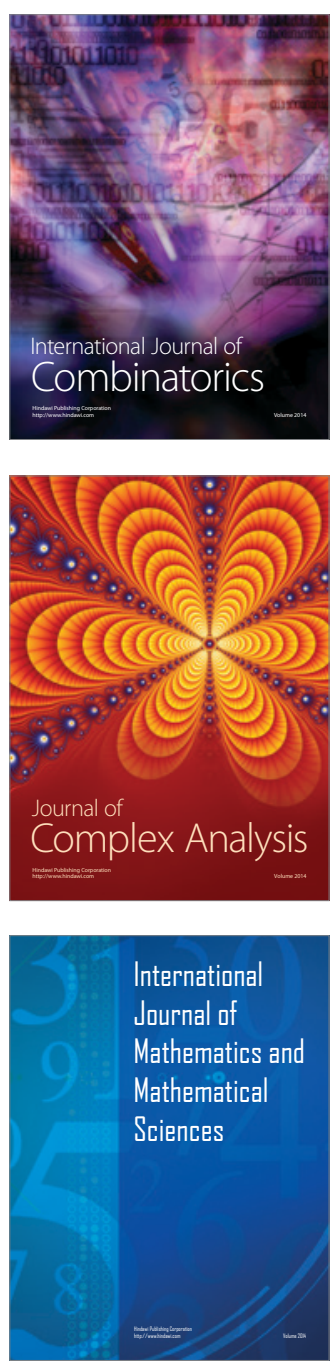
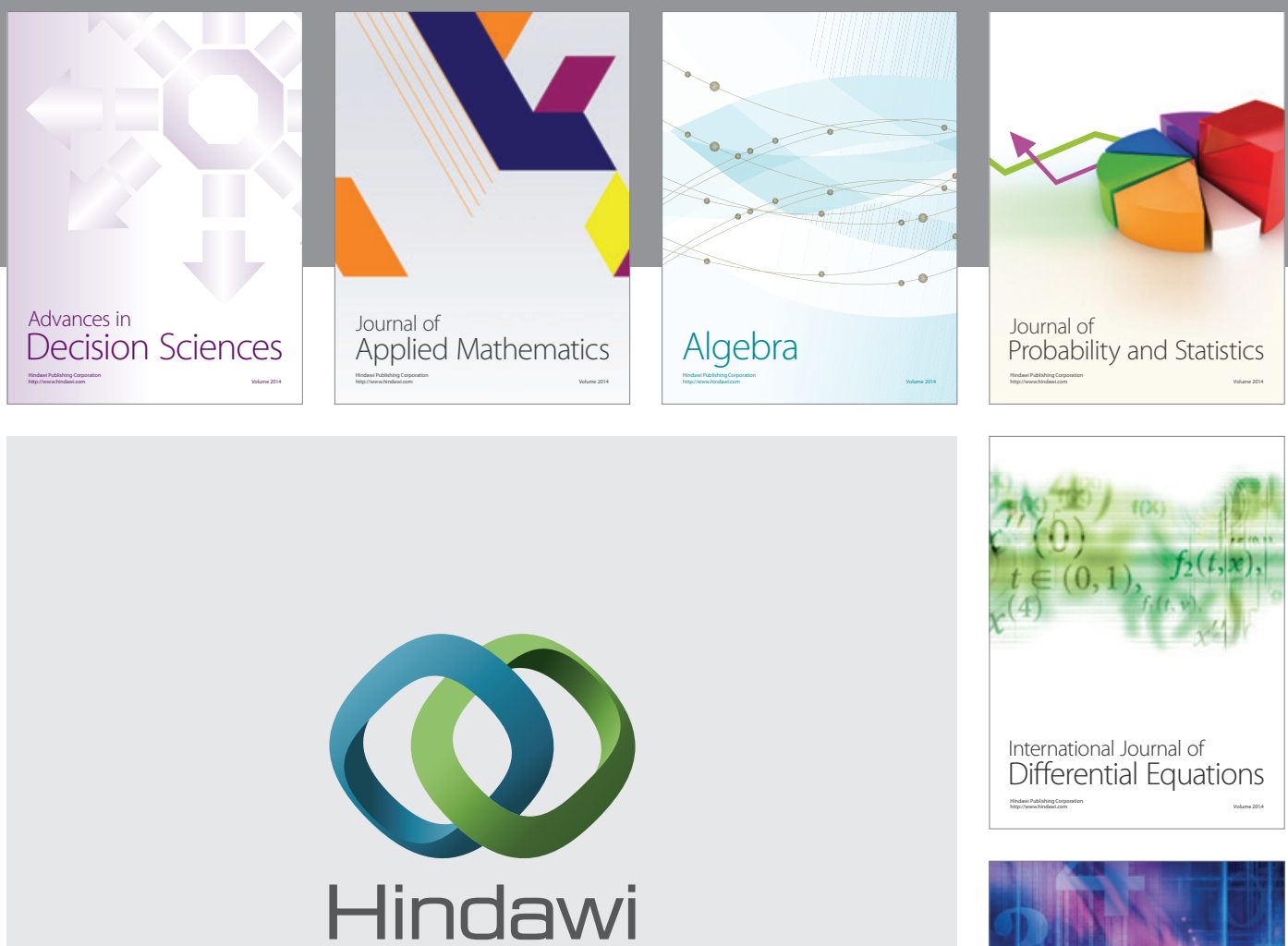

Submit your manuscripts at http://www.hindawi.com
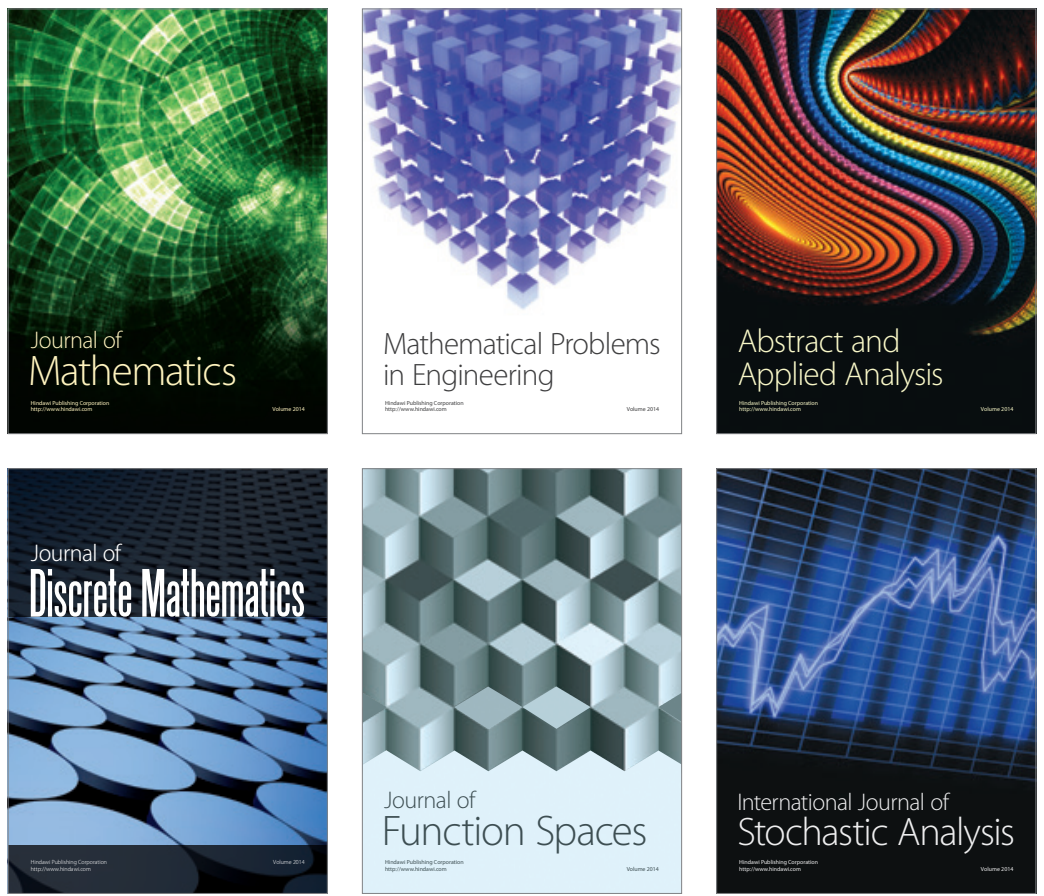

Journal of

Function Spaces

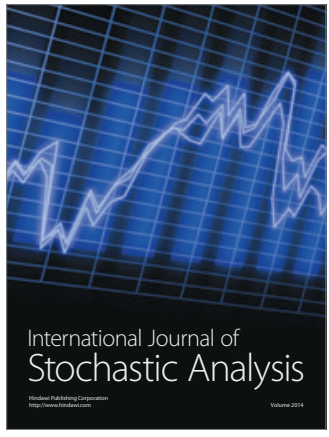

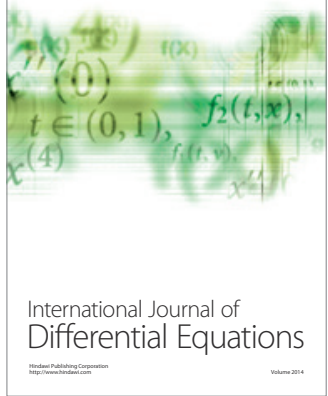
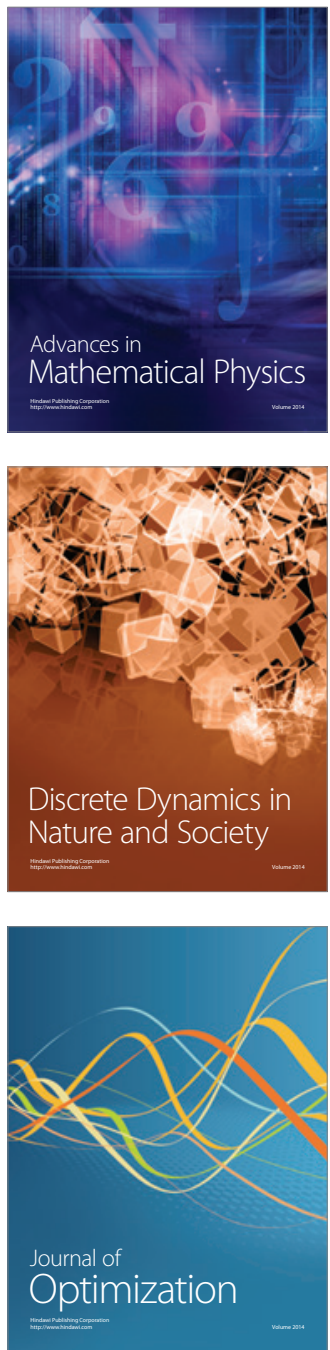